\title{
A randomized controlled trial of Mindfulness-Based Cognitive Therapy (MBCT) versus treatment-as-usual (TAU) for chronic, treatment-resistant depression: study protocol
}

Mira B. Cladder-Micus ${ }^{1,2,3^{*}}$, Janna N. Vrijsen ${ }^{2,3}$, Eni S. Becker ${ }^{1}$, Rogier Donders ${ }^{4}$, Jan Spijker ${ }^{1,2}$ and Anne E. M. Speckens ${ }^{3}$

\begin{abstract}
Background: Major depression is a common psychiatric disorder, frequently taking a chronic course. Despite provision of evidence-based treatments, including antidepressant medication and psychological treatments like cognitive behavioral therapy or interpersonal therapy, a substantial amount of patients do not recover. Mindfulness-Based Cognitive Therapy (MBCT) has been found to be effective in reducing relapse in recurrent depression, as well as lowering symptom levels in acute depression. The effectiveness of MBCT for chronic, treatment-resistant depression has only be studied in a few pilot trials. A large randomized controlled trial is necessary to examine the effectiveness of $\mathrm{MBCT}$ in reducing depressive symptoms in chronic, treatment-resistant depression.

Methods/Design: A randomized-controlled trial is conducted to compare MBCT with treatment-as-usual (TAU). Patients with chronic, treatment-resistant depression who have received antidepressant medication and cognitive behavioral therapy or interpersonal therapy are included. Assessments take place at baseline and post intervention/ TAU-period. The primary outcome are depressive symptoms. Secondary outcomes are: remission rates, quality of life, rumination, mindfulness skills and self-compassion. Patients in the TAU condition are offered to participate in the MBCT after the post TAU-period assessment. From all completers of the MBCT (MBCT condition and patients participating after the TAU-period), follow-up assessments are taken at three and six months after the completion of the MBCT.
\end{abstract}

Discussion: This trial will result in valuable information about the effectiveness of MBCT in chronic, treatment-resistant depressed patients who previously received antidepressant medication and psychological treatment.

Trial registration: trialregister.nl NTR4843, registered 14 $4^{\text {th }}$ October 2014.

Keywords: Mindfulness-Based cognitive therapy, Chronic depression, Treatment-resistant depression, Randomized-controlled trial, Depressive symptoms, Rumination, Quality of life

\footnotetext{
*Correspondence: m.cladder-micus@psych.ru.nl

'Behavioural Science Institute, Radboud University, Nijmegen, The

Netherlands

${ }^{2}$ Pro Persona Expertisecentrum Depressie, Institute for Mental Health Care,

Nijmegen, The Netherlands

Full list of author information is available at the end of the article
}

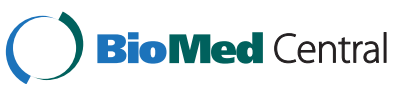

(c) 2015 Cladder-Micus et al. Open Access This article is distributed under the terms of the Creative Commons Attribution 4.0 International License (http://creativecommons.org/licenses/by/4.0/), which permits unrestricted use, distribution, and reproduction in any medium, provided you give appropriate credit to the original author(s) and the source, provide a link to the Creative Commons license, and indicate if changes were made. The Creative Commons Public Domain Dedication waiver (http://creativecommons.org/publicdomain/zero/1.0/) applies to the data made available in this article, unless otherwise stated. 


\section{Background}

Major depression is one of the most common psychiatric disorders. It has a large impact on individual's wellbeing and leads to high societal costs $[1,2]$. The duration of a depressive episode is highly variable. Whereas the majority of people in the general population with a major depressive episode recovers within several months, approximately $20 \%$ of all depressed patients develop a chronic course of depression [2,3]. The latter is associated with higher suicidality [4], increased comorbidity with other psychiatric disorders, and impaired physical and psychosocial functioning [5]. According to DSM-IV criteria, a depressive episode is defined as chronic if the depressive episode persists for at least two years [6]. However, results of a large prospective epidemiological study on the prevalence of major depression in the Dutch population show, that -even if treated in mental health care- the rate of recovery decreases after a few months. After one year, recovery rates virtually stagnate, meaning that if no recovery has taken place within 12 months the probability to develop a chronic course of depression- as defined in the DSM-IV- increases substantially [3].

Not only a chronic course of symptoms but also several unsuccessful therapeutic interventions can be indicative of a severe depressive episode. Patients who do not respond to treatment are often described as 'treatment-resistant'. However, up till now, an unified and clear definition of 'treatment-resistant depression' is lacking. Definitions range from one single unsuccessful attempt at antidepressant medication (ADM) to several pharmacological interventions combined with electroconvulsive therapy $[7,8]$. In most clinical studies, a depressive episode is regarded as treatment-resistant when successive treatment with two antidepressants has not led to a significant improvement of depressive symptoms. Interestingly, this definition does not take response to psychological treatment into account, while cognitive behavior therapy (CBT) or interpersonal therapy (IPT) are part of international treatment guidelines for recurrent and chronic depression [9]. Importantly, research has shown that even when psychological and pharmacological treatment options are combined, a substantial amount of patients fails to respond $[10,11]$. This means that there is a dire need of new treatment options for chronic, treatment resistant depression.

In recent years, mindfulness based interventions have been developed as a treatment focused on distress caused by long-lasting somatic and psychological disorders [12]. Mindfulness is defined as non-judgmental awareness of the present moment [13] and was introduced by Kabat-Zinn [14] as a treatment option for patients with chronic somatic conditions. Segal, Williams and Teasdale [15] adapted the program for patients with recurrent depression, naming it 'Mindfulness Based
Cognitive Therapy' (MBCT). MBCT is a group intervention, consisting of eight weekly $2.5 \mathrm{~h}$ sessions and a silent day of $6 \mathrm{~h}$. It involves meditation exercises as well as cognitive behavioral techniques. MBCT is designed to address processes that are associated with the onset, maintenance and recurrence of depression, such as maladaptive automatic cognitive and behavioral patterns in reaction to negative emotions or thoughts. In first instance participants are taught to allow and acknowledge negative emotions and thoughts as they are, without having judgments about them or reacting to them. In this way, they start recognizing their own maladaptive automatic patterns. By creating a greater awareness of habitual behavioral and cognitive patterns (e.g. avoidance or rumination) participants learn to deliberately disengage from them. Another important part of MBCT is to develop selfcompassion. MBCT differs from CBT in the sense that the focus of cognitive therapy is more on the content of thoughts, while MBCT focuses on the process of thinking.

In chronic depression, maladaptive automatic cognitive and behavioral patterns often have become habitual. Patients often experienced several episodes of depression and an early onset of the disorder. To learn to react in a different way to these maladaptive automatic cognitive and behavioral patterns and to develop a non-judgmental awareness of depressive symptoms might be especially useful for this population. Therefore MBCT might be effective in lowering depressive symptoms in chronic, treatment resistant depression.

A recent meta-analysis [16] shows that MBCT is effective in preventing relapse in patients with recurrent depression. Additionally, there is evidence, that MBCT not only decreases chance of relapse of depression, but that it lowers levels of residual or current depressive symptoms. The first studies investigating the effect of MBCT on residual symptoms in remitted depressed patients found significant improvements of depressive symptoms $[17,18]$. More recently, a meta-analysis was conducted on the effects of MBCT in patients currently meeting diagnostic criteria for a major depressive disorder [19]. The authors concluded that MBCT reduces depressive symptoms in currently depressed patients and might be as effective as CBT. One of the studies [20] included in the meta-analysis specifically investigated possible differences of the effectiveness of MBCT between remitted but still symptomatic and currently depressed patients. The results indicate that MBCT was effective for both remitted and currently depressed patients.

First results indicate that MBCT might also be a promising treatment option for chronic depression. Kenny and Williams [21] reported a decrease in depressive symptoms in a sample of currently depressed patients who experienced symptoms for one year or longer, preceded by three or more previous episodes. In line with that, Eisendrath et 
al. [22] found a significant decrease in depressive symptoms after MBCT in currently depressed patients who previously received at least two different antidepressants before starting the $\mathrm{MBCT}$ training. In addition to these nonrandomized studies, two randomized-controlled pilot trials demonstrated a significant reduction of depressive symptoms in chronically depressed patients. Barnhofer et al. [23] found that after following MBCT less participants met full criteria for a major depressive episode compared to a control group. Another study [24] investigated the effectiveness of person-based cognitive therapy, a therapy combining mindfulness techniques and cognitive therapy, in chronic depression and found significant improvement of depressive symptoms and mindfulness skills in the therapy group compared to the control group. Importantly, all of these studies report a good acceptance of the MBCT training procedures by chronically depressed patients.

In sum, there is preliminary evidence that MBCT might be effective in reducing depressive symptoms in chronic, treatment-resistant patients. However well-powered randomized controlled trials are necessary to systematically examine the effectiveness of MBCT compared to treatment as usual in this severely affected patient group. With the current ongoing trial we will hopefully contribute to the knowledge about treatment options for patients with persistent depressive symptoms. Taking into account the high risk for chronic depression if symptoms persist for more than one year [3] we will recruit patients experiencing a depressive episode for 12 months or longer who underwent previous pharmacological and psychological treatment.

\section{Aims}

The aim of the current study is to investigate the effect of MBCT compared to treatment as usual (TAU) on depressive symptoms in patients with a current depressive episode persisting for at least 12 months, who previously received pharmacological treatment, as well as either CBT or IPT. We hypothesize that patients who receive MBCT in addition to TAU will report lower levels of depressive symptoms, higher quality of life, and less rumination, compared to TAU alone. In addition, we expect that mindfulness skills and self-compassion will improve in the MBCT condition. Possible effects of level of treatmentresistance and experience of childhood trauma on treatment effect will also be investigated.

\section{Methods/Design}

\section{Design}

This study is a multi-center open-labeled randomized controlled trial with two groups: MBCT+ TAU vs. TAU. The study has been approved by the Medical Ethics Committee Arnhem-Nijmegen (nr. 2012/339) for all participating sites. Before randomization, written informed consent is obtained from all participants. Primary and secondary outcome measures are assessed at baseline and after MBCT + TAU (8-12 weeks) or after a TAUperiod (8-12 weeks). Participants allocated to the TAU condition are offered participation in the MBCT after completion of the TAU-period and first follow-up assessment. Of all participants who completed the MBCT, follow-up measures take place at three and six months after the end of treatment. For an overview of the recruitment and measurement points see Fig. 1.

\section{Participants}

The study population consist of outpatients with a current depressive episode lasting for 12 months or longer who previously received ADM, as well as either CBT or IPT. Patients are recruited at a university medical center (Radboudumc Centre for Mindfulness) and at different locations of a regional mental health institution (Pro Persona) in The Netherlands. Patients can be referred by health care professionals, or can contact the research team themselves for participation. Patients receive an information letter and are contacted by phone one week later by the research team. Patients are informed that participation in the study is completely voluntary and that they can withdraw from the study at any time. Participants are free to receive additional treatment or to change their (dose of) pharmacological medication during the study period.

\section{Inclusion criteria:}

- Age $\geq 18$

- A current diagnosis of major depressive disorder according to DSM-IV criteria with a duration of $\geq 12$ months

- Moderate to high level of current depressive symptoms (Inventory of Depressive Symptomatology- Self Report, IDS-SR $\geq 21$ )

- At least one adequate trial of ADM during the current episode (defined as: appropriate doses of antidepressant medication for $\geq 4$ weeks; or patient's refusal to use medication in contrast to advise by a psychiatrist)

- Previous psychological treatment during the current episode (defined as: $\geq 10$ sessions of CBT or IPT; or $<10$ sessions if discontinued because of patient's withdrawal)

\section{Exclusion criteria:}

- Current psychotic symptoms

- Bipolar disorder according to DSM-IV

- Current alcohol or drugs dependence according to DSM-IV

- Recent electro convulsive therapy (<3 months)

- Current somatic disorder (partly) explaining depressive symptoms 
- Physical-, language-, cognitive-, or intellectual impairments which interfere with participation in MBCT or assessments

- Previous MBCT training

\section{Procedure}

Assessment of eligibility, baseline measures and randomization

All patients who indicated their interest in the study are invited for a research interview. Written informed consent is obtained during this first appointment. To assess eligibility and to classify comorbid psychiatric disorders, participants are interviewed by a research psychologist or psychiatrists in training with the depression, anxiety, psychosis and addiction modules of the Mini International Neuropsychiatric Interview (MINI; [25]). In addition, the following disorder-related characteristics are assessed: duration of current major depressive episode, number of psychological treatment sessions (i.e. CBT and/or IPT) during current major depressive episode, duration and doses of pharmacological treatments during the current depressive episode, number of depressive episodes during lifetime and age of onset. On the base

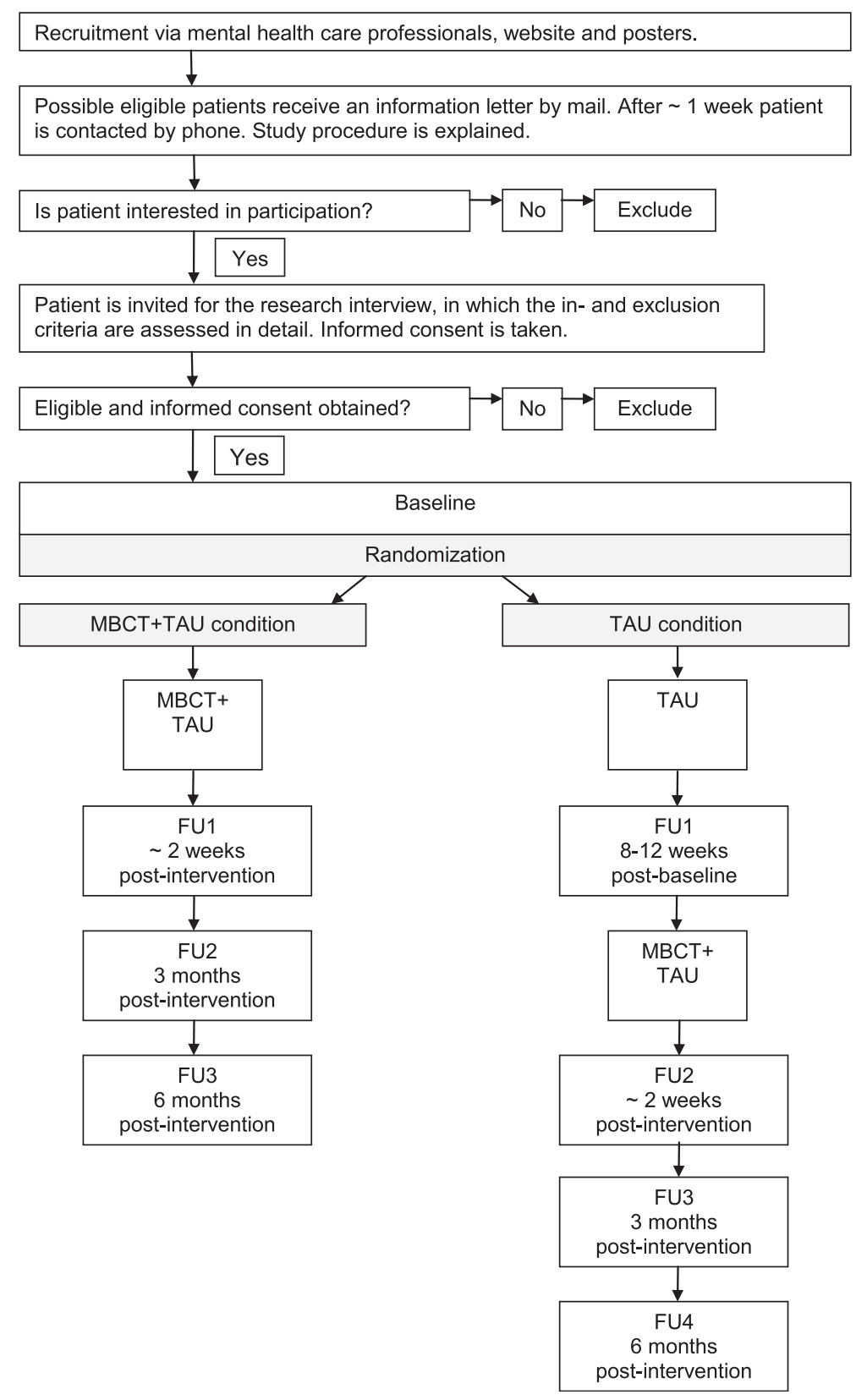

Fig. 1 Flowchart of the recruitment and measurement points 
of these details the research assistant fills out the Dutch Measure for quantification of Treatment Resistant Depression (DM-TRD, [26] [27]). After completion of the baseline questionnaires, a computer program is used to randomly allocate patients to either MBCT + TAU or TAU. A minimization strategy is used for severity of symptoms (IDS-SR $\geq 21$ and $\leq 31$ vs. $>31$ and $\leq 39$ vs. $>39$ ) and duration of major depressive episode (1-2 years vs. $>2$ years). If the period between randomization and start of the MBCT training exceeds four weeks in the MBCT + TAU condition, a repeated baseline assessment takes place.

\section{Follow-up assessments}

The first follow-up measure takes place after the MBCT training or the TAU-period. The MINI depression module is administered face-to-face or by phone and participants fill out the questionnaires either online or on paper. Participants are asked about changes in pharmacological or psychological treatment during the study period. Also participants deciding to discontinue MBCT are asked to participate in follow-up measures. In all MBCT completers (MBCT + TAU and participants of the TAU group who followed the MBCT after the TAU-period), the same follow-up measures are administered at three and six months after end of treatment. Due to logistic reasons it is not possible to keep assessors blind. To assess the inter-rater reliability of the MINI, all diagnostic interviews are audio taped and a randomly selected sample will be reassessed by an independent rater. For an overview of all measurement assessments see Table 1.

Table 1 Overview of measures and corresponding measurement time points

\begin{tabular}{lllllll}
\hline Measure & Target concept & Baseline & FU1 & FU2 & FU3 & FU4 \\
\hline IDS-SR & Depressive symptoms & $\bullet$ & $\bullet$ & $\bullet$ & $\bullet$ & $\bullet$ \\
MINI-depr. & Diagnosis of major & $\bullet$ & $\bullet$ & $\bullet$ & $\bullet$ & $\bullet$ \\
module & depression & & & & & \\
WHOQOL- & Quality of life & $\bullet$ & $\bullet$ & $\bullet$ & $\bullet$ & $\bullet$ \\
BREF & & & & & \\
RRS-EXT & Rumination & & $\bullet$ & $\bullet$ & $\bullet$ & $\bullet$ \\
FFMQ & Mindfulness skills & $\bullet$ & $\bullet$ & $\bullet$ & $\bullet$ & $\bullet$ \\
SCS & Self-compassion & $\bullet$ & $\bullet$ & $\bullet$ & $\bullet$ & $\bullet$ \\
MINI & Comorbid psychiatric & $\bullet$ & & & & \\
interview & disorders & & & & \\
DM-TRD & Treatment-resistance & $\bullet$ & & & \\
CTQ & Childhood trauma & $\bullet$ & & &
\end{tabular}

FU1: 2 weeks post intervention (MBCT + TAU)/8-12 weeks post baseline (TAU), FU2: 3 months post intervention (MBCT+ TAU)/2 weeks post intervention (TAU), FU3: 6 months post intervention $(M B C T+T A U) / 3$ months post intervention(TAU), FU4: 6 months post intervention (TAU)

\section{Interventions}

MBCT + TAU

The MBCT consists of eight weekly sessions of $2.5 \mathrm{~h}$ in groups of 8-15 patients and one additional day of silent practice between the $5^{\text {th }}$ and $7^{\text {th }}$ session. Participants are asked to do daily homework assignments. MBCT combines mindfulness meditation techniques, as sitting meditation and the body scan with elements of cognitive therapy such as psycho education and the identification of automatic negative thoughts. The MBCT manual described by Segal, Williams and Teasdale [13] is used. Participants are enrolled in regular MBCT trainings provided at the Radboudumc Centre for Mindfulness and Pro Persona for (remitted) depressed patients. This means that the training groups not only consist of study participants but also of regular patients with a wide range of depressive symptoms.

MBCT trainings are taught by experienced mindfulness trainers who completed a 2-year post-graduate teacher training program at the Radboudumc Centre for Mindfulness. With this, they all meet the training criteria described in the MBCT good practice guidelines (UK Mindfulness-Based Teacher Trainer Network, 2011). Two MBCT sessions of each trainer are videotaped to verify adherence to the MBCT protocol. In addition to MBCT the participants receive TAU.

\section{TAU}

TAU may consist of any treatment the participant or current mental health care specialist sees as necessary. Participants are encouraged to continue treatment they followed prior to enrolling in the study. The number of treatment sessions and use of antidepressant medication are recorded.

\section{Measurements \\ Primary outcome measure}

\section{Depressive symptoms}

The primary outcome measure is the level of depressive symptoms measured with the Inventory of Depressive Symptomatology Self-Report (IDS-SR). The IDS-SR is a 30-item self report questionnaire and has good psychometric properties [28]. The IDS-SR has previously been used in research on MBCT in depression and has been shown to be sensitive to change [17].

\section{Secondary outcome measures}

\section{Remission rates}

Remission from depression according to DSM-IV criteria is assessed with the major depression module of the Mini International Neuropsychiatric Interview (MINI; [25]). 


\section{Quality of life}

Perceived quality of life is measured using the 26 -item World Health Organization Quality of Life scale (WHOQOL-BREF; [29]). Psychometric properties were tested in a sample of Dutch psychiatric outpatients and are considered to be good [30].

\section{Rumination}

Rumination is measured with the extended version of the Ruminative Response Scale (RRS-EXT [31, 32]. The RRS-EXT distinguishes between a more reflective way of thinking (i.e. reflective pondering) and a more maladaptive way of thinking about symptoms (i.e. brooding) [33].

\section{Mindfulness skills}

Mindfulness skills are measured with the Five Facets Mindfulness Questionnaire (FFMQ. [34]. The FFMQ consists of 39 items which are divided in five subscales: observing, describing, acting with awareness, nonjudging of inner experience and non-reactivity of inner experience. The Dutch version of the FFMQ has good psychometric properties $[35,36]$ and has previously been used in research on mindfulness skills in bipolar disorder [37].

\section{Self-compassion}

Self-compassion is assessed with the Self Compassion Scale (SCS; $[38,39])$. The SCS is an 26-item self report measure and consists of six components: self-kindness, self-judgment, common humanity, isolation, mindfulness, and over-identification. The scale has good psychometric properties and high scores on the SCS are related to psychological well-being [39].

\section{Possibly moderating variables}

\section{Treatment-resistance}

The Dutch Measure for quantification of Treatment Resistant Depression (DM-TRD; $[26,27]$ ) is used to investigate treatment-resistance of the current depressive episode. With the DM-TRD several dimensions of treatment-resistance are scored by the clinician (duration, symptom severity, functional impairment, comorbid anxiety symptoms, prosocial stressors, treatment failures of pharmacological and psychological treatment) which leads to a total score ranging from 2-26.

\section{Childhood trauma}

To assess the experience of traumatic childhood events, participants fill out the Childhood Trauma Questionnaire (CTQ-SF; [40]). This 28-item questionnaire measures traumatic experiences and maltreatment during childhood. The CTQ-SF has previous been validated in a Dutch clinical sample [41].

\section{Data handling}

All study-related information is stored in secure folders with limited access. Paper-based data collection forms only contain participant numbers to maintain participant confidentiality and are stored in a locked cabinet in an area with limited access. Electronic data files are password protected. The list linking participant number and personal information is stored in a separated password protected file. Paper-based data entry will be double checked. Data will be stored for 15 years after the end of inclusion. The study has low to negligibly risks therefore no data monitoring committee is assigned. Only the first author and principal investigators or persons assigned by them will have access to the final data set. Authors of the final trial report will have made substantial contribution to the design, conduction, interpretation and reporting of the trial. We will not use professional writers. Study results will be presented via publications and presentations; study participants, funders and involved clinicians will receive a summary of the study results.

\section{Statistical analyses \\ Power analysis and sample size}

A power analysis was executed based on the findings of van Aalderen et al. [20]. Van Aalderen and colleagues [20] reported an effect size of 0.53 (Cohen's $d$ ) in a sample of 69 currently depressed patients. Based on an alpha of 0.05 and power of $80 \%$, estimating the correlation between baseline and first follow-up measurements to be 0.5 and intending to use an ANCOVA controlling for baseline levels of depression, a needed sample size of 43 per condition was calculated. Expecting a drop-out of 8 patients, a total of 94 participants will be recruited.

\section{Analyses plan}

The MBCT + TAU and TAU conditions will be compared on baseline sample characteristics to ensure that all possible meaningful characteristics were evenly distributed across the conditions. To examine treatment outcome, we will use both intention-to-treat and per-protocol samples. Participants are considered completers of the MBCT if they attend at least four sessions of MBCT. To examine whether effects for participants experiencing a depressive episode for one to two years and participants meeting criteria for chronic depression according to the DSM-IV $(\geq 2$ years) are different, the primary and secondary analyses will be repeated in these subgroups.

\section{Primary analysis}

The effect of condition (MBCT + TAU vs. TAU) on the primary outcome measure (depressive symptoms) will be examined using general linear models. Condition and baseline score of depressive symptoms will be included as independent variables, depressive symptoms at FU1 
as the dependent variable. Because MBCT is delivered in mixed groups consisting of regular patients and a few study participants and previous studies did not yield an effect of therapy group [20], we will not incorporate a random group effect.

\section{Secondary analyses}

Effects on quality of life, rumination, mindfulness skills, and self-compassion will also be investigated using general linear models with the same independent variable as in the primary analysis. To investigate effects on remission rates a $X^{2}$ test will be performed. Moderating effects of level of treatment-resistance and childhood trauma will be investigated by including an interaction effect (condition"moderator) in the models. Furthermore, exploratory analyses on rumination, mindfulness skills and self-compassion as possible underlying mechanisms of change will be conducted. Consolidation and moderation of treatment effect will be investigated in the whole sample of MBCT completers (MBCT + TAU and TAU) using end-of-treatment assessments and follow-up assessments at three and six months after end of treatment.

\section{Discussion}

Despite the range of currently available treatments options such as antidepressant medication and cognitive behavioral or interpersonal therapy, a substantial number of currently depressed patients do not recover [10]. Chronic, treatment-resistant depression is associated with a high personal as well as societal burden $[4,5]$. Additional treatment options are required, to further reduce depressive symptoms and to improve quality of life in this population.

In previous pilot studies, MBCT appeared to be a promising treatment option for patients with chronic depression. Besides incorporating effective elements of cognitive behavioral therapy, MBCT focuses on acknowledging negative emotions and thoughts and disengaging from maladaptive automatic cognitive and behavioral patterns elicited by them. As chronic, treatment-resistant depression is characterized by perseverance of symptoms and a long treatment history, maladaptive automatic thinking patterns are likely to have become habitual. Therefore MBCT might be a very valuable additional treatment for this group. In the current study we will build upon the previous pilot findings by studying the effect of MBCT on depressive symptoms in a large chronic, treatmentresistant sample in a randomized controlled trial.

Strauss and colleagues [19] argue that MBCT might be as effective as cognitive behavioral therapy in reducing depressive symptoms in currently depressed patients. The results of the current study will provide an indication of the effectiveness of MBCT over and above available pharmacological and psychological treatments such as CBT and IPT, in patients who previously received these types of psychological treatment.

In addition to the effects of MBCT on depressive symptoms, we will investigate if MBCT leads to less rumination and higher quality of life in this patient population. Moreover, this trial will inform clinicians and researchers if chronic, treatment-resistant depressed patients are able to learn mindfulness skills and to develop more self-compassion by following a standard MBCT training. This large trial will also give more detailed information about the acceptability and effect sizes of $\mathrm{MBCT}$ in this target group.

The ecological validity of the study will be high, as participants will be enrolled in standard Dutch MBCT courses offered at mental health care institutions. If the conclusion will be that MBCT is effective in reducing depressive symptoms in chronic, treatmentresistant depression, this study could make a significant impact on treatment options for this severely depressed target group.

\section{Abbreviations \\ ADM: Antidepressant medication; CBT: Cognitive behavioral therapy; CTQ-SF: Childhood Trauma Questionnaire-short form; DM-TRD: Dutch measure of treatment resistant depression; DSM-IV: Diagnostic and statistical manual of mental disorders- $4^{\text {th }}$ edition; FFMQ: Five facet mindfulness questionnaire; IDS-SR: Inventory of Depressive Symptomatology- Self Report; IPT: Interpersonal therapy; MBCT: Mindfulness-based Cognitive Therapy; MINI: Mini International Neuropsychiatric Interview; RRS-EXT: Ruminative response scale extended version; SCS: Self compassion scale; TAU: Treatment as usual; WHOQoL-BREF: World health organization quality of life scale}

\section{Competing interests}

The authors declare that they have no competing interests.

\section{Authors' contributions}

All authors contributed to the design of the study. AS and JS are the principal investigators of the study. MCM drafted the manuscript, which was added to and modified by JV, EB, AS and JS. RD specifically contributed to the power analysis and statistical analyses plan. MCM coordinates data collection and recruitment of participants. All authors read and approved the final manuscript.

\section{Acknowledgements}

The current research is funded by Fonds Psychische Gezondheid, Netherlands Foundation for Mental Health (grant no. 2011 6630). The funder is not involved in data collection, analyses, interpretation of results, writing the report or the decision to submit the manuscript.

\section{Author details}

'Behavioural Science Institute, Radboud University, Nijmegen, The Netherlands. ${ }^{2}$ Pro Persona Expertisecentrum Depressie, Institute for Mental Health Care, Nijmegen, The Netherlands. ${ }^{3}$ Department of Psychiatry, Radboud university medical centre, Nijmegen, The Netherlands. ${ }^{4}$ Department for Health Evidence, Radboud university medical centre, Nijmegen, The Netherlands.

Received: 16 September 2015 Accepted: 13 October 2015

Published online: 09 November 2015

\section{References}

1. De Graaf R, Ten Have M, Van Dorsselaer S. De psychische gezondheid van de Nederlandse bevolking. Utrecht: Nemesis-2: Opzet en eerste resultaten, Trimbos-Instituut; 2010. 
2. Kessler RC, Berglund P, Demler O, et al. The epidemiology of major depressive disorder: results from the national comorbidity survey replication (ncs-r). JAMA. 2003;289(23):3095-105. doi:10.1001/jama.289.23.3095.

3. Spijker J, De Graaf R, Bijl RV, Beekman AT, Ormel J, Nolen WA. Duration of major depressive episodes in the general population: results from the Netherlands Mental Health Survey and Incidence Study (NEMESIS). Br J Psychiatry. 2002;181(3):208-13.

4. Spijker J, de Graaf R, ten Have M, Nolen WA, Speckens A. Predictors of suicidality in depressive spectrum disorders in the general population: results of the netherlands mental health survey and incidence study. Soc Psychiatry Psychiatr Epidemiol. 2010;45(5):513-21.

5. Blanco C, Okuda M, Markowitz JC, Liu S-M, Grant BF, Hasin DS. The epidemiology of chronic major depressive disorder and dysthymic disorder: results from the national epidemiologic survey on alcohol and related conditions. J Clin Psychiatry. 2010;71(12):1645.

6. American Psychiatric Association. Diagnostic and statistical manual of mental disorders, text revision (DSM-IV-TR). American Psychiatric Association; 2000. Washington, DC

7. Berlim MT, Turecki G. What is the meaning of treatment resistant/refractory major depression (TRD)? A systematic review of current randomized trials. Eur Neuropsychopharmacol. 2007:17(11):696-707.

8. Birkenhäger TK, Ruhé HG. The Pharmacological Frontiers in Treatment Resistant Major Depression. Psychiatric Disorders-New Frontiers. In: Affective Disorders. 2013. p. 41.

9. NICE. Depression: the Treatment and Management of Depression in Adults (Update). vol 2009. NICE clinical guideline 90. 2009.

10. Torpey DC, Klein DN. Chronic depression: update on classification and treatment. Current Psychiatry Reports. 2008;10(6):458-64.

11. Spijker J, van Straten A, Bockting C, Meeuwissen J, van Balkom A. Psychotherapy, antidepressants, and their combination for chronic major depressive disorder: a systematic review. Can J Psychiatry. 2013;58(7):386-92.

12. Khoury B, Lecomte T, Fortin G, Masse $M$, Therien $P$, Bouchard $V$, et al. Mindfulness-based therapy: a comprehensive meta-analysis. Clin Psychol Rev. 2013;33(6):763-71.

13. Segal, Williams, Teasdale. Mindfulness-based cognitive therapy for depression. Guilford Press; 2012.

14. Kabat-Zinn J. Full catastrophe living. New York: How to cope with stress, pain and illness using mindfulness meditationDell; 1990.

15. Segal Z, Williams JG, Teasdale JD. Mindfulness-based cognitive therapy for depression: A new approach to preventing relapse. New York: Guilford; 2002.

16. Piet J, Hougaard E. The effect of mindfulness-based cognitive therapy for prevention of relapse in recurrent major depressive disorder: a systematic review and meta-analysis. Clin Psychol Rev. 2011;31(6):1032-40. doi:10.1016/ j.cpr.2011.05.002

17. Geschwind N, Peeters F, Huibers M, van OS J, Wichers M. Efficacy of mindfulness-based cognitive therapy in relation to prior history of depression: randomised controlled trial. Br J Psychiatry. 2012;201(4):320-5. doi:10.1192/bjp.bp.111.104851.

18. Kingston T, Dooley B, Bates A, Lawlor E, Malone K. Mindfulness - based cognitive therapy for residual depressive symptoms. Psychol Psychother Theory Res Pract. 2007;80(2):193-203.

19. Strauss C, Cavanagh K, Oliver A, Pettman D. Mindfulness-based interventions for people diagnosed with a current episode of an anxiety or depressive disorder: a meta-analysis of randomised controlled trials. PLoS One. 2014;9(4), e96110.

20. van Aalderen JR, Donders ART, Giommi F, Spinhoven P, Barendregt HP, Speckens AEM. The efficacy of mindfulness-based cognitive therapy in recurrent depressed patients with and without a current depressive episode: a randomized controlled trial. Psychol Med. 2011;42(05):989-1001. doi:10.1017/s0033291711002054.

21. Kenny MA, Williams JMG. Treatment-resistant depressed patients show a good response to mindfulness-based cognitive therapy. Behav Res Ther. 2007;45(3):617-25. doi:10.1016/j.brat.2006.04.008.

22. Eisendrath SJ, Delucchi K, Bitner R, Fenimore P, Smit M, McLane M. Mindfulness-based cognitive therapy for treatment-resistant depression: a pilot study. Psychother Psychosom. 2008;77(5):319-20. doi:10.1159/ 000142525

23. Barnhofer $T$, Crane $C$, Hargus $E$, Amarasinghe $M$, Winder $R$, Williams JMG. Mindfulness-based cognitive therapy as a treatment for chronic depression: a preliminary study. Behav Res Ther. 2009;47(5):366-73. doi:10.1016/ j.brat.2009.01.019.
24. Strauss C, Hayward M, Chadwick P. Group person-based cognitive therapy for chronic depression: a pilot randomized controlled trial. Br J Clin Psychol. 2012;51(3):345-50

25. Overbeek T, Schruers K, Griez E. MINI: Mini International Neuropsychiatric Interview, Dutch version 5.0. 0 (DSM-IV). Maastricht: University of Maastricht; 1999.

26. Ruhé HG, van Rooijen G, Spijker J, Peeters FP, Schene AH. Staging methods for treatment resistant depression. A systematic review. J Affect Disord. 2012;137(1):35-45

27. Spijker J. Chronisch depressief in nieuw perspectief. SI: sn. 2013.

28. Rush AJ, Gullion CM, Basco MR, Jarrett RB, Trivedi MH. The inventory of depressive symptomatology (IDS): psychometric properties. Psychol Med. 1996:26(3):477-86.

29. Skevington SM, Lotfy M, O'Connell KA. The World Health Organization's WHOQOL-BREF quality of life assessment: psychometric properties and results of the international field trial. A report from the WHOQOL group. Qual Life Res. 2004;13(2):299-310.

30. Trompenaars FJ, Masthoff ED, Van Heck GL, Hodiamont PP, De Vries J. Content validity, construct validity, and reliability of the WHOQOL-Bref in a population of Dutch adult psychiatric outpatients. Qual Life Res. 2005;14(1):151-60.

31. Treynor W, Gonzalez R, Nolen-Hoeksema S. Rumination reconsidered: a psychometric analysis. Cogn Ther Res. 2003;27(3):247-59.

32. Raes F, Hermans D. The revised version of the Dutch ruminative response scale. 2007. http://www.ekgp.ugent.be/pages/nl/vragenlijsten/RRS-NL.pdf.

33. Schoofs $H$, Hermans D, Raes F. Brooding and reflection as subtypes of rumination: evidence from confirmatory factor analysis in nonclinical samples using the dutch ruminative response scale. J Psychopathol Behav Assess. 2010;32(4):609-17.

34. Baer RA, Smith GT, Lykins E, Button D, Krietemeyer J, Sauer S, et al. Construct validity of the five facet mindfulness questionnaire in meditating and nonmeditating samples. Assessment. 2008;15(3):329-42.

35. de Bruin El, Topper M, Muskens JG, Bögels SM, Kamphuis JH. Psychometric properties of the Five Facets Mindfulness Questionnaire (FFMQ) in a meditating and a non-meditating sample. Assessment. 2012;19(2):187-97.

36. Veehof MM, Peter M, Taal E, Westerhof GJ, Bohlmeijer ET. Psychometric properties of the Dutch Five Facet Mindfulness Questionnaire (FFMQ) in patients with fibromyalgia. Clin Rheumatol. 2011;30(8):1045-54.

37. Deckersbach T, Hölzel BK, Eisner LR, Stange JP, Peckham AD, Dougherty DD, et al. Mindfulness-based cognitive therapy for nonremitted patients with bipolar disorder. CNS NeuroSci Therapeutics. 2012;18(2):133-41.

38. Neff KD. The development and validation of a scale to measure self-compassion. Self Identity. 2003;2(3):223-50.

39. Neff KD, Vonk R. Self-compassion versus global self-esteem: Two different ways of relating to oneself. J Pers. 2009;77(1):23-50

40. Bernstein DP, Stein JA, Newcomb MD, Walker E, Pogge D, Ahluvalia T, et al. Development and validation of a brief screening version of the childhood trauma questionnaire. Child Abuse Negl. 2003;27(2):169-90. doi:10.1016/ s0145-2134(02)00541-0

41. Thombs BD, Bernstein DP, Lobbestael J, Arntz A. A validation study of the dutch childhood trauma questionnaire-short form: factor structure, reliability, and known-groups validity. Child Abuse Negl. 2009;33(8):518-23. doi:10.1016/j.chiabu.2009.03.001

\section{Submit your next manuscript to BioMed Central and take full advantage of:}

- Convenient online submission

- Thorough peer review

- No space constraints or color figure charges

- Immediate publication on acceptance

- Inclusion in PubMed, CAS, Scopus and Google Scholar

- Research which is freely available for redistribution 